\title{
2 Akutschmerztherapie und postoperative Schmerztherapie bei adipösen Patienten
}

\author{
Christoph H.R. Wiese und Christoph L. Lassen
}

In den meisten deutschen Kliniken sind Anästhesisten mit einem etablierten Akutschmerzdienst in die Behandlung von Patienten mit Schmerzen eingebunden. Zum weit überwiegenden Anteil findet diese Behandlung in der postoperativen Phase (komplexe Akutschmerztherapie entsprechend OPS Ziffer 8-919) und als konsiliarärztliche Tätigkeit statt. Daher befasst sich dieses Kapitel im Wesentlichen mit der postoperativen (bzw. perioperativen) Akutschmerztherapie und deren Besonderheiten bei adipösen Patienten. Andere Formen der Akutschmerztherapie (z.B. Schmerzen nach Unfällen oder bei akuten Erkrankungen) werden analog bei normalgewichtigen und adipösen Patienten durchgeführt.

\subsection{Grundsätze der perioperativen Schmerztherapie}

Die individuelle und differenzierte postoperative Schmerztherapie bei adipösen Patienten beginnt idealerweise schon in der präoperativen Phase, wird in der operativen und postoperativen Phase fortgesetzt und endet erst mit Entlassen des Patienten sowie einer entsprechenden Empfehlung an den hausärztlichen Kollegen in der poststationären Phase. Sie wird somit immer als perioperative Gesamtschmerztherapie betrachtet. Der Analgetikabedarf ist individuell unterschiedlich und in entsprechender Rücksprache mit den besonderen Bedürfnissen sowohl des Patienten als auch der weiteren Behandler umzusetzen und im Verlauf zu kontrollieren und ggf. anzupassen.

\section{Grundsätze im Einzelnen}

- Die perioperative Schmerztherapie orientiert sich an Patientenfaktoren (z.B. Vormedikation, Vorerkrankungen) und an operativen Faktoren (Art des Eingriffs, Zugangsweg).

- Die Kombination verschiedener Medikamente und verschiedener Therapieverfahren ist erstrebenswert, um das Nebenwirkungspotenzial einzelner Substanzen zu reduzieren.

- Statt Verzicht auf Analgetika wegen möglicher Nebenwirkungen muss eine ausreichende und antizipatorische Therapie (z.B. Obstipationsprophylaxe) erfolgen. Die möglichen Nebenwirkungen sollten mit dem Patienten im Vorfeld besprochen und ihm auch Optionen zu ihrer Behandlung aufgezeigt werden.

- Analgetika können und sollten möglichst zeitnah im Verlauf der Therapie neben der i.v. Gabe oral verabreicht werden. Insbesondere die intramuskuläre aber auch die subkutane Applikation sind im Rahmen der Akutschmerztherapie zu vermeiden.

\subsection{Gründe für perioperative Schmerztherapie}

Alle Patienten haben das Recht, eine suffiziente Schmerztherapie zu erhalten. Unbehandelte Schmerzen können zur Folge haben: 
1. erhöhte Morbidität und Mortalität

2. verlängerten Intensivaufenthalt

3. insgesamt längere Klinikverweildauer

Zusätzlich erhöht sich statistisch signifikant das Risiko einer Schmerzchronifizierung bei unbehandelten postoperativen Schmerzen (Burke u. Shorten 2009). Eine gute schmerztherapeutische Versorgung reduziert mögliche Ängste des Patienten und führt zu einer deutlich erhöhten Patientenzufriedenheit (Burke u. Shorten 2009). Es sind im Sinne der Vorteile der schmerztherapeutischen Versorgung besonders die indirekten Vorteile für eine Klinik von entscheidender Bedeutung, da sich die OPS Ziffer 8-919 nicht direkt als vergütungsrelevant erweist.

\subsection{Spezielle Situation bei adipösen Patienten}

Die perioperative Schmerztherapie bei adipösen Patienten stellt den behandelnden Arzt vor die Herausforderung, besonders darauf zu achten, dass durch die Analgetika keine schwerwiegenden Nebenwirkungen hervorgerufen werden (Bennett et al. 1982). Besonders gefürchtet ist vor allem bei der Patientenversorgung auf einer Normalpflegestation die Gefahr der Atemdepression durch Opioid-Analgetika. Diese kann vor allem bei der Dosierung nach absolutem Körpergewicht und bei Vorliegen anderer Risiken, insbesondere dem obstruktiven Schlaf-Apnoe-Syndrom (OSAS), auftreten. Eine Monotherapie mit Opioid-
Analgetika sollte in diesem Zusammenhang vermieden werden, und weiterhin sollte die Medikationsdosierung nicht an das Gesamtkörpergewicht der Patienten angepasst, sondern an der Wirkung adaptiert werden. Zusätzlich empfiehlt es sich, statt einer Monotherapie eine sehr differenzierte multidimensionale Schmerzmedikation zu nutzen (sogenannte multimodale Analgesie).

Unter multimodaler Analgesie versteht man das Einbeziehen verschiedener Therapieformen (u.a. medikamentöse Therapie, psychologische Schmerztherapie, Physiotherapie) aber auch in einzelnen Definitionen die Kombination mehrerer unterschiedlicher analgetisch wirksamer Präparate in die Schmerztherapie. Hierzu gehören beispielsweise die systemische Analgesie mit Opioid-Analgetika und Nicht-Opioid-Analgetika, die lokale, regionale und neuroaxiale Analgesie, die Gabe verschiedener Medikamente zur präemptiven Analgesie, die Co-Analgetika und die adjuvante medikamentöse Therapie.

\subsection{Systemische Medikamentengabe}

Je nach Intensität der postoperativen Schmerzen kommen in der Therapie verschiedene Analgetika zum Einsatz. Hierbei erfolgt die Auswahl der Medikamente nach Intensität und Art des Schmerzes, sowie Patientenfaktoren (Allergien, Vorerkrankungen, Risiko für Nebenwirkungen). Hierbei stellt das WHO-Stufenschema, obwohl primär für die Tumor-

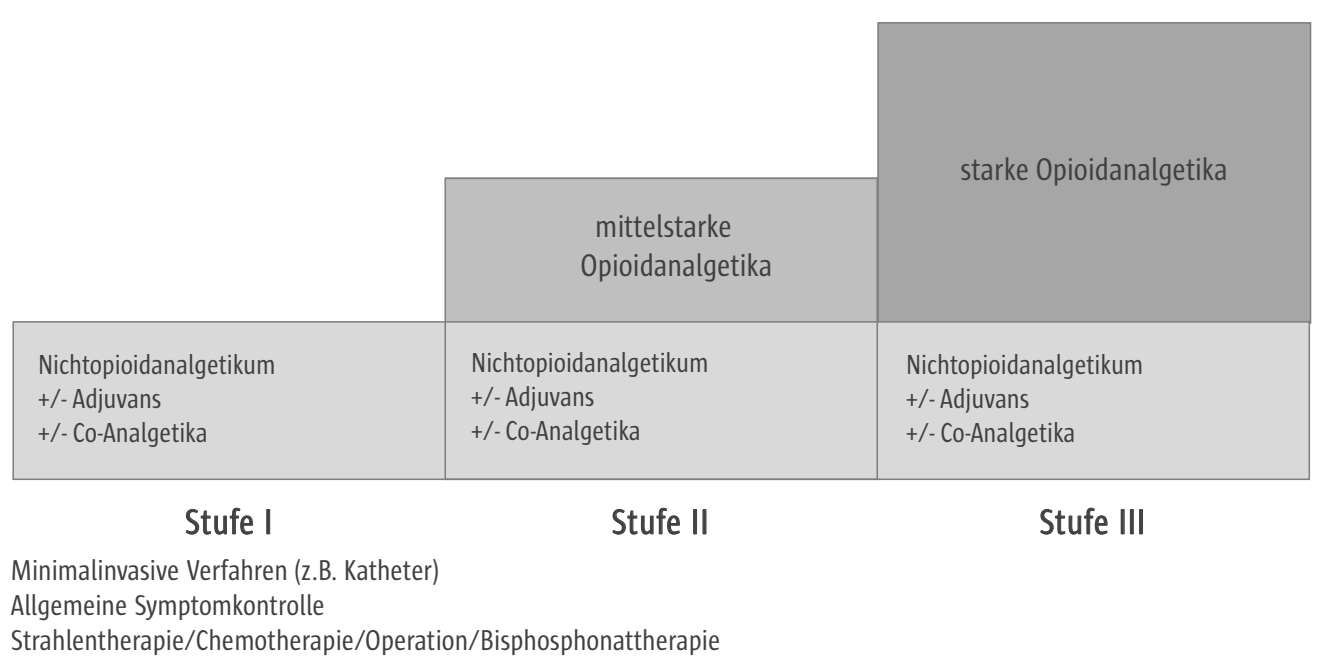

Abb. 1 WHO-Stufenschema (modifiziert nach WHO 1986) 
schmerztherapie entwickelt, auch die Basis in der postoperativen Schmerztherapie dar. Oftmals wird jedoch mit der Stufe III begonnen und die Therapie im Verlauf entsprechend reduziert, sodass auch vom „umgekehrten“ 2 -Stufen-Schema der Akutschmerztherapie gesprochen werden kann. Die Stufenauswahl bezieht sich ausschließlich auf die Intensität des Schmerzes und lässt andere Faktoren in der Regel außer Acht (s. Abb. 1).

Im Folgenden sollen die verschiedenen medikamentösen Möglichkeiten dargestellt und vor dem speziellen Hintergrund ihres Einsatzes bei adipösen Patienten diskutiert werden.

\subsubsection{Opioide}

Opioide stellen im Rahmen der postoperativen Schmerztherapie die am häufigsten gebrauchte Substanzklasse bei mittlerer bis starker Schmerzintensität dar. Opioide lassen sich in unterschiedlichsten Formen (z.B. intravenöse, orale Gabe, retardierte, schnell wirksame Form) verabreichen. In der unmittelbaren postoperativen Phase im Aufwachraum stellt die intravenöse Gabe die am einfachsten durchzuführende Applikationsart und somit den Standard dar. Hier erfolgt die Gabe bei adipösen wie bei normalgewichtigen Patienten in mehreren Einzeldosen an den individuellen Patientenbedarf adaptiert. Bei der Verlegung aus dem Aufwachraum ist bei adipösen Patienten auf einen ausreichenden Wachheitsgrad zu achten, im Zweifel sollte eine verlängerte Überwachung im Aufwachraum erfolgen. Im Einzelfall ist eine weitere engmaschige Kontrolle der Vitalparameter und ggf. Monitorüberwachung auf der Normalpflegestation für die ersten 24 Stunden anzustreben. Auch Patienten mit OSAS sollten im Aufwachraum eine adäquate, bedarfsadaptierte Schmerztherapie bekommen. Dies schließt die Gabe von Opioiden ein. Aufgrund des erhöhten Risikos für postoperative Apnoephasen empfehlen manche Autoren auch bei diesen Patienten eine Überwachung zumindest der Sauerstoffsättigung über Nacht auf der Normalpflegestation (Ostermeier et al. 1997).

Die orale Gabe von retardierten Opioiden zur Basisanalgesie gewinnt zunehmend an Bedeutung. Sie wird empfohlen für Patienten nach mittelgroßen Eingriffen und zur Fortführung der analgetischen Therapie nach großen Eingriffen, wenn die Patienten Medikamente per os aufnehmen können (Pogatzki-Zahn u. Zahn 2007). Bei Schulung aller Mitarbeiter können diese Medikamente auch auf der Normal- pflegestation sicher und effektiv eingesetzt werden. Spezielle Untersuchungen zur Sicherheit bei adipösen Patienten fehlen jedoch derzeit noch, sind aber bei der zunehmenden Problematik der Adipositas in Deutschland für die Zukunft anzustreben.

Vielfach untersucht dagegen ist die Sicherheit der Anwendung von patienten-kontrollierten intravenösen Analgesieverfahren (PCIA). Hier liegen auch Untersuchungen zur Sicherheit der PCIA bei adipösen Patienten vor (Choi et al. 200o), wobei die Aussagekraft dieser Studie durch die kleine Anzahl der beobachteten Patienten deutlich limitiert ist und somit auch in dieser Fragestellung weitere Arbeiten notwendig erscheinen.

\section{Orale Schmerztherapie/PCIA auf Normalpflegestation}

In unserer Klinik setzen wir orale Opioide und PCIA auf Normalpflegestation auch bei adipösen Patienten regelhaft ein. Insbesondere bei der Anwendung der PCIA sind wir großzügig mit der Überwachung der Sauerstoffsättigung. Aus unserer Sicht sollte diese bei jedem Patienten mit diagnostiziertem OSAS erfolgen, u.a. auch aus medikolegalen Gründen. Wir verwenden die gleichen Standardeinstellungen der PCIA wie bei jedem anderen Patienten, eine Erhöhung der Bolusdosis nur aufgrund des Körpergewichtes sollte nicht erfolgen.

Nicht empfohlen werden können andere Applikationsformen wie die transdermale, intramuskuläre und subkutane Gabe von Opioiden. Die transdermale Applikation von Opioiden mittels Pflaster ist langsam und konstant und trägt damit der häufig wechselnden Schmerzintensität postoperativ keine Rechnung. Außerdem ist die Aufnahme des Opioids in den Kreislauf bei Adipösen aufgrund größerer subkutaner Fettschichten zusätzlich verlangsamt und die generelle Abgabe und deren Beurteilung generell beeinträchtigt. Intramuskuläre und subkutane Injektionen sollten aufgrund der unvorhersehbaren Verstoffwechselung der Medikamente bei adipösen Patienten perioperativ nicht erfolgen.
Cave: Transdermale, intramuskuläre und subkutane Applikation von Opioiden sind bei adipösen Patien- ten ungünstig. 


\subsubsection{Nicht-Opioid-Analgetika}

Nicht-Opioid-Analgetika stellen einen wichtigen Grundstein in der Behandlung von perioperativen Schmerzen dar. Sie führen zu einer Reduktion der benötigten Opioiddosen und damit zu einer Reduktion des Risikos von Opioid-induzierten Nebenwirkungen (Rocca et al. 2005). Dieses Einsparen von Opioiden und die sich daraus ergebenden Vorteile in der postoperativen Phase (durch ein geringeres Nebenwirkungsspektrum) konnte auch bei Patienten mit Adipositas gezeigt werden (Meyer 2002).

Die Auswahl der Nicht-Opioid-Analgetika erfolgt streng nach Kontraindikationen und orientiert sich dann an Art des operativen Eingriffs:

Bei Knochen- und Weichteilschmerzen wird der Einsatz klassischer und selektiver nicht-steroidaler Antirheumatika (NSAR) präferiert.

Bei viszeralen Schmerzen kommt insbesondere in Deutschland Metamizol zum Einsatz. Die schmerztherapeutische Rolle und Bedeutung von Paracetamol, vor allem in der intravenösen Zubereitung, ist noch umstritten (Remy et al. 2006).

\subsubsection{Perioperative Gabe sogenannter "Co-Analgetika“}

Die perioperative Gabe von Medikamenten, die nicht klassischerweise unter die Gruppe der Analgetika fallen, hat in den letzten Jahren an Bedeutung gewonnen. Mehrere Substanzen wurden hierbei mit zum Teil widersprüchlichen Studienergebnissen untersucht. Dazu gehört ein Teil der Gruppe der Antikonvulsiva (beispielsweise Gabapentin, Pregabalin), das Ketamin/S-Ketamin, Magnesium, Dexmedetomidin/Clonidin und intravenöses Lidocain.

In einer Studie bei Patienten, die mit einem Magenbypass versorgt wurden, wurde intraoperatives Fentanyl mit einer intraoperativen Analgesie durch Ketamin, Magnesium, Methylprednisolon, Ketorolac, Clonidin und Lidocain verglichen (Feld et al. 2003). Hierbei zeigte sich eine gleiche Schmerzreduktion bei niedrigerem postoperativem Morphinbedarf in der Kombinationsgruppe.

Inwieweit eine solche Kombination außerhalb von kontrollierten Studienbedingungen im klinischen Alltag einsetzbar ist, bliebe zu diskutieren. Viel versprechend erscheint jedoch der Einsatz von Dexmedetomidin, einem $\alpha_{2}$-Agonisten, der in Deutschland aktuell zur Zulassung kommt (Tufanogullari et al. 2008). Interessant ist in diesem Zusammenhang auch eine weitere klinische Untersuchung, die eine Reduktion der postoperativen Morphingabe im Aufwachraum mittels einer kontrollierten Aromatherapie mit Lavendel erreichen konnte (Kim et al. 2007).

Die Nutzung der Co-Analgesie ist insbesondere bei adipösen Patienten von großem Interesse, da durch eine effektive Schmerzreduktion in der Kombinationstherapie auch der Gesamtbedarf an Opioid-Analgetika reduziert und damit auch deren Nebenwirkungen vermindert werden können.

\subsection{Infiltrationsanalgesie}

Die Infiltration von chirurgischen Wunden mit Lokalanästhetika unmittelbar vor Verschluss der Haut ist ein einfaches und probates Mittel zur Reduktion des unmittelbaren Wundschmerzes und damit auch der direkten postoperativen Opioidmedikation. Über die Einlage eines Katheters kann eine definierte Menge von Lokalanästhetikum auch postoperativ weiter appliziert werden. Dieses Konzept wird erleichtert durch spezielle Katheter mit mehreren Öffnungen, über die das Lokalanästhetikum auch bei längeren Hautschnitten alle Bereiche der Wunde erreicht. Das Prinzip der Wundinfiltration wurde auch bei adipösen Patienten getestet und mit anderen Analgesieverfahren verglichen (Schumann et al. 2003). Es zeigte sich, dass die Gruppe mit Wundinfiltration + PCIA zwar der Cruppe mit Epiduralanalgesie unterlegen, der Cruppe mit alleiniger PCIA aber überlegen war und somit insbesondere bei adipösen Patienten mögliche Nebenwirkungen der Opioid-Analgetika reduziert werden konnten.

\subsection{Periphere Regionalanästhesie}

Die periphere Regionalanästhesie bietet gegenüber einer Allgemeinanästhesie insbesondere bei adipösen Patienten einige Vorteile, unter anderem eine gute postoperative Analgesiequalität unter Verzicht auf die Gabe von Opioiden bzw. deren Reduktion.

Die Anlage einer peripheren Regionalanästhesie ist bei Adipösen jedoch technisch erschwert. Aufgrund der breiteren subkutanen Fettschicht sind gerade die tief liegenden Strukturen schwieriger zu erreichen (z.B. N. ischiadicus), als die relativ nah an der Hautoberfläche liegenden Nerven (z.B. Plexus brachialis). 
Hilfreich kann der Einsatz von Ultraschall zur genaueren Lokalisation der relevanten Strukturen sein, da sogenannte „Landmarken" gerade bei ausgeprägter Adipositas nur schwierig zu bestimmen sind. Aber auch mit Ultraschall sind nur oberflächliche Strukturen sicher darstellbar und dann leichter zu erreichen (Schwemmer et al. 2006). Tiefe Strukturen können auch mit Ultraschall unter Umständen gar nicht oder nur nach aufwändiger Lagerung identifiziert werden (Chantzi et al. 2007).

Trotz der schwierigeren technischen Durchführbarkeit sollte eine Regionalanästhesie (ggf. als Zusatz zur Allgemeinanästhesie) auch bei ausgeprägter Adipositas angestrebt werden, um so die systemischen Nebenwirkungen der Opioid-Analgetika zu reduzieren.

\subsection{Neuroaxiale Regionalanästhesie}

Für die neuroaxiale Regionalanästhesie gelten ähnliche Grundsätze wie für die periphere. Auch hier kann die postoperative Analgesie unter Verzicht auf systemische Opioide gestaltet werden. Gleichzeitig gilt jedoch auch, dass die Anlage eines neuroaxialen Anästhesieverfahrens bei sehr ausgeprägter Adipositas erschwert sein kann. Die wichtigste „Landmarke“ bei neuroaxialen Punktionen stellen die Dornfortsätze der Wirbelsäule dar. Diese sind bei manchen Patienten nicht durch Tasten zu identifizieren.

Häufig ist für die spinale/epidurale Punktion eine längere Nadel als sonst gebräuchlich notwendig.

In manchen Fällen kann zur Identifikation des Epiduralraums die Sonografie verwendet werden (Balki et al. 2009).

Die Anwendung der thorakalen Epiduralanästhesie bei adipösen Patienten ist vergleichsweise gut untersucht. Unter anderem konnte in Studien gezeigt werden, dass hierdurch der postoperative Morphinbedarf und die pulmonale Komplikationsrate gesenkt werden konnten.

Die Gabe von epiduralen Opioiden verbessert zwar die Qualität der Schmerztherapie, sollte aber bei Patienten mit OSAS unterbleiben, bzw. nur unter Monitorüberwachung stattfinden (Schumann et al. 2009).
Praxisbeispiel: Schmerztherapie bei adipösen Patienten für Abdominaloperationen

Ziel der perioperativen Schmerztherapie ist ein adäquates Analgesieniveau mit geringem Nebenwirkungs/Komplikationsrisiko. Dies kann wie dargestellt auf verschiedenen Wegen erreicht werden. In unserer Einrichtung führen wir, wenn möglich, eine thorakale Epiduralanalgesie durch. Postoperativ bleibt der Periduralkatheter je nach Situation 4-7 Tage in situ. Nach Entfernen des PDK wird die Schmerztherapie mit retardiertem oralen Opioid, einem kurzwirksamen Opioid bei Bedarf und einem NichtOpioid-Analgetikum (vorzugsweise Metamizol) weitergeführt.

Sollte die Anwendung einer Epiduralanalgesie kontraindiziert sein oder seitens des Patienten abgelehnt werden, empfehlen wir zur Anästhesieeinleitung Lidocain als Bolus i.v. $(1,5 \mathrm{mg} / \mathrm{kgKG} \mathrm{IBW})$ sowie danach kontinuierlich per Perfusor intra- und postoperativ über einen Zeitraum von vier Stunden ( $2 \mathrm{mg} / \mathrm{kgKG} \mathrm{IBW} / \mathrm{h}$ ). Alternativ wird zum Wundverschluss die Wunde mit langwirksamem Lokalanästhetikum durch den Operateur infiltriert (z.B. 20-30 ml Ropivacain 0,75\%). Die Schmerztherapie auf der Normalpflegestation erfolgt mittels Piritramid-PCIA und NichtOpioid-Analgetikum (z.B. Metamizol 1,25 g i.v. alle 6 h). Eine Umstellung auf orale Medikamentengabe wird frühestmöglich angestrebt und durchgeführt.

\section{Zusammenfassung}

Zusammenfassend kann gesagt werden, dass sich die Akutschmerztherapie bei adipösen Patienten nicht wesentlich von der bei normalgewichtigen Patienten unterscheidet. Prinzipiell sollte aber die medikamentöse Therapie an die Wirkung und nicht an das absolute Gewicht des Patienten adaptiert werden. Der Einsatz von Co-Analgetika und Regionalverfahren ist besonders beim adipösen Patienten zu befürworten, um den postoperativen Bedarf an Opioid-Analgetika weitestgehend reduzieren zu können und hierdurch die bei adipösen Patienten möglicherweise gehäuft auftretenden Nebenwirkungen dieser Substanzklasse zu verringern.

\section{Literatur}

Balki M, Lee Y, Halpern S, Carvalho JC (2009) Ultrasound imaging of the lumbar spine in the transverse plane: the correlation between estimated and actual depth to the epidural space in obese parturients. Anesth Analg 108, 1876-1881

Bennett R, Batenhorst R, Graves DA, Foster TS, Griffen W0, Wright BD (1982) Variation in postoperative analgesic requirements in the morbidly obese following gastric bypass surgery. Pharmacotherapy $2,50-53$ 
Burke S, Shorten GD (2009) When pain after surgery doesn't go away. Biochem Soc Trans 37, 318-322

Chantzi C, Saranteas T, Zogogiannis |, Alevizou N, Dimitriou V (2007) Ultrasound examination of the sciatic nerve at the anterior thigh in obese patients. Acta Anaesthesiol Scand 51, 132

Choi YK, Brolin RE, Wagner BK, Chou S, Etesham S, Pollak P (2000) Efficacy and safety of patient-controlled analgesia for morbidly obese patients following gastric bypass surgery. Obes Surg 10 , 154-159

Feld JM, Laurito CE, Beckerman M, Vincent I, Hoffman WE (2003) Non-opioid analgesia improves pain relief and decreases sedation after gastric bypass surgery. Can I Anaesth 50, 336-341

Kim JT, Ren C), Fielding GA, Pitti A, Kasumi T, Wajda M, Lebovits A, Bekker A (2007) Treatment with lavender aromatherapy in the post-anesthesia care unit reduces opioid requirements of morbidly obese patients undergoing laparoscopic adjustable gastric banding. Obes Surg 17, 920-925

Meyer R (2002) Rofecoxib reduces perioperative morphine consumption for abdominal hysterectomy and laparoscopic gastric banding. Anaesth Intensive Care 30, 389-390

Ostermeier AM, Roizen MF, Hautkappe M, Klock PA, Klafta JM (1997) Three sudden postoperative respiratory arrests associated with epidural opioids in patients with sleep apnea. Anesth Analg 85 , $452-460$
Pogatzki-Zahn EM, Zahn PK (2007) Neue Möglichkeiten der systemischen Analgesie. Anasthesiol Intensivmed Notfallmed Schmerzther 42, 22-31

Remy C, Marret E, Bonnet F (2006) State of the art of paracetamol in acute pain therapy. Curr Opin Anaesthesiol 19, 562-565

Rocca GD, Chiarandini P, Pietropaoli P (2005) Analgesia in PACU: nonsteroidal anti-inflammatory drugs. Curr Drug Targets 6, 781-787

Schumann R, Jones SB, Cooper B, Kelley SD, Bosch MV, Ortiz VE, Connor KA, Kaufman MD, Harvey AM, Carr DB (2009) Update on best practice recommendations for anesthetic perioperative care and pain management in weight loss surgery, 2004-2007. Obesity (Silver Spring) 17, 889-894

Schumann R, Shikora S, Weiss JM, Wurm H, Strassels S, Carr DB (2003) A comparison of multimodal perioperative analgesia to epidural pain management after gastric bypass surgery. Anesth Analg 96, 469-474

Schwemmer U, Papenfuss T, Greim C, Brederlau |, Roewer N (2006) Ultrasound-guided interscalene brachial plexus anaesthesia: differences in success between patients of normal and excessive weight. Ultraschall Med 27, 245-250

Tufanogullari B, White PF, Peixoto MP, Kianpour D, Lacour T, Griffin I, Skrivanek G, Macaluso A, Shah M, Provost DA (2008) Dexmedetomidine infusion during laparoscopic bariatric surgery: the effect on recovery outcome variables. Anesth Analg 106, 1741-1748

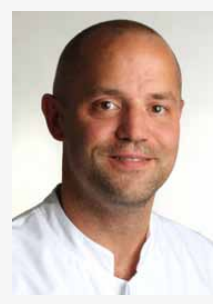

\section{PD Dr. med. Christoph H.R. Wiese}

Nach dem Medizinstudium an der Georg-Augustin-Universität Göttingen Beginn der klinischen Tätigkeit 1999 am Klinikum Braunschweig in der Klinik für Anästhesiologie. Von 20022008 Arzt in Weiterbildung am Uniklinikum Göttingen. 2002 Promotion. Ab 2004 Facharzt für Anästhesiologie und Erlangung der Zusatzbezeichnungen Notfallmedizin, Intensivmedizin, spezielle Schmerztherapie und Palliativmedizin. Seit 2009 Oberarzt der Klinik für Anästhesiologie am Uniklinikum Regensburg sowie Leiter der interdisziplinären Schmerzambulanz. 2010 Habilitation zum Thema „Palliative Notfälle - eine neue Herausforderung für die Notfallmedizin“.

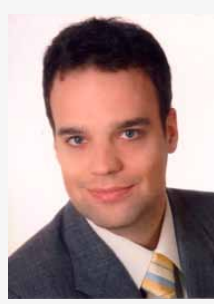

\section{Dr. med. Christoph L. Lassen}

Nach dem Medizinstudium an der Christian-Albrechts-Universität Kiel mit Forschungsaufenthalt an der University of Utah in Salt Lake City, USA, arbeitete er seit 2005 als Arzt in Weiterbildung am Universitätsklinikum Regensburg in der Klinik für Anästhesiologie. 2009 Promotion. Besondere Interessensschwerpunkte sind Schmerztherapie und Palliativmedizin. 